\title{
Verhandlungen der Royal Society of Medicine.
}

\author{
Dermatologische Abteilung.
}

Sitzung vom 20. April 1911.

Davis, H. Cheilitis exfoliativa bei 18jähr. Mädchen von anämischem Aussehen, mit Seborrhoe und Menstruationsanomalien. Der Zustand begann vor 4 Jahren mit Rhagaden an den Unterlippen, es folgte Bil-: dung von Krusten, die sich abstoben, um neuen Platz zu machen. An der Oberlippe nur leichte Einrisse, Schuppen, keine Krusten. Beabsichtigte Behandlung: Röntgenstrahlen.

Diskussion. M or ris $M$. hat das Leiden mit schwarzer Zunge: vergesellschaftet gesehen. Radium dürfte besser wirken als Röntgenstrahlen. Little G. erinnert an einen ganz ähnlichen von ihm vorgestellten Fall (1909); Zinkionisation scheint wirksam. Mac L e od hat einen gleichartigen Fall in Behandlung, bei welchem Röntgenstrablen wirkungslos waren. Eine ähnliche, vielleicht seborrhoische Affektion der Lippen mit Verdickung und Schuppung reagiert auf Röntgenstrahlen gut.

Fearnsides. Fall von funktionellem hysteris chem Troph ödem. 16jähr. Knabe; eigene und Familienanamnese ohne Besonderheiten. Vor einem halben Jahre drang ihm ein Nagel eines Kistendeckels vom Handrücken ber tief 'in den ersten Interkostalraum der rechten Hand. Heftiger Schmerz, noch am gleichen Tage auffallende Schwäche der Hand, am nächsten Tage Schwellung des Daumens, später der ganzen Hand. Dieser Zustand dauert seitdem trotz mancherlei Behandlung in wechselndem Grade an, bei Tage zunehmend, bei Nacht zurückgehend. Die aktiven Bewegungen der Finger sind durch die Schwellungen behindert, sonst normal. Sensibilität vollständig erhalten. Charakteristische Anästhesie des Gaumens. Unter festem Pflasterverband, der Einflußnahme seitens des P. selbst ausschließt, hat neuerdings die Schwellung stark abgenommen. Der Fall ist aufzufassen als ein solcher von Jokaler Lymphgefäßlähmung psychischen Ursprungs; er spricht für die Ba binski sche Deutung ähnlicher Zustände.

Diskussion. W e ber $P$. verweist auf die reiche einschlägige Literatur des Auslandes.

Little, G. 1. Herpes z os ter der l. Hälfte der Stirne und des Haarbodens bei 50jähr. Manne. Das l. Auge durch Schwellung beider Lider vollständig verdeckt, aber auch das r. Unterlid stark geschwollen. Kein Schmerz im Gegensatze zu dem gewöhnlichen Verhalten des Herpes 
zoster gerade in diesem Gebiete. Die Schwellung der Unterlider bei so typischem Zoster des 1. Trigeminusastes scheint überhaupt noch nicht beschrieben zu sein.

Diskussion. Morris M. Ist in solchen Fällen auch der Nasenast des Nervs befallen, so ist das Auge in Gefahr. Redner berichtet über einen Parallelfall zu dem vorliegenden: die Lider beider Augen zu gleicher Zeit einbezogen, übrigens lebhafter Schmerz. S e qu ei $\mathbf{r}$ a betrachtet die Lidschwellung der Gegenseite als einfache Folge der Lymphgefäßanastomose.

2. 13jähr. anämischer Junge mit Eryth e ma ab igne. Er fröstelte in den letzten 3 Wochen und saß viel am Feuer, wobei er die Hände auf die Knie zu legen pflegte. Jetzt an beiden Unterschenkelvorderflächen gefensterte Pigmentation; die Maschen des Netzes auffallenderweise erhaben und fast warzig, was mit einer Verdickung der Haut an den Knien zusammenhängen könnte. Die Handrücken, besonders der rechte, ebenso gezeichnet, doch ohne Erhabenheit der pigmentierter Haut. Die Hautveränderungen müssen sich erstaunlich rasch entwickelt haben.

Diskussion. Morris M. glaubt nicht, daß sich ein solcher Zustand in 3 Wochen heransbilden könne. Zuweilen sind ähnliche Bilder auf Stauung und Kälte zurückzuführen. Whitfield sieht die Epidermisverdickung als nahe verwandt mit Angiokeratoma an: zuerst entsteht wohl chronische Kongestion mit Teleangiektasien, auf diesem Boden dann der warzige Zustand. W eber P. pflichtet dem Vorstellenden in der Erklärung der Hauterscheinungen vollkommen bei.

Mac Leod, J. M. H. F a 11 z u r D i a g n o s e. 50jähr. Frau mit Narben am Halse als einzigem Anzeichen von Tbk.; eine Schwester an Tbk. gestorben. Im Gesichte d. P. flache, bis guldengroße, wohl umschriebene, nicht druckschmerzhafte Hautverdickungen, anzufühlen wie Gummi elasticum; entsprechend den älteren von ihnen ist die Haut etwas vorgewölbt und leicht gerötet mit kleinen Teleangiektasien. Sie baben Neigung spontan zu verschwinden unter Hinterlassung einer kleinen Lücke ausschließlich im Unterhautgewebe. Finsenlicht beschleunigt den Räckgang. Von B esniers Lupus erythematodes mit tiefen Schwellungen unterscheidet sich die Affektion dadurch, daß ihr Ausgangspunkt im subkutanen Gewebe liegt; die Veränderungen des Derma entstehen sekundär, zum Teile durch den Druck von unten, gerade wie in einem dem Vortragenden bekannten Falle von Paraffineinspritzung. Die wahrscheinlichste Auffassung: Skrofulodermen ohne Aufbruch. Biopsie nicht zugelassen. Ein ähnlicher Fall ist als noduläre Tuberkulose des Hypoderma“ kürzlich von $L$. W e n de veröffentlicht worden.

Dlskussion. Morris M. Solche Grübchen bleiben bei gewissen erythema-induratumartigen Veränderungen an den Beinen zurück. Dore hat einen Fall von Lupus erythematodes gesehen, der sklerodermatisch wurde. Little A. hat in der Londoner dermatologischen Gesellschaft im Februar 1901 einen Fall vorgestellt, welcher mit dem vorliegenden Ähnlichkeit hat. Die Herde saßen am Arm. Später stellte sich Lupus vulgaris bei dem Kranken ein. Sequeira betont die Übereinstimmung mit dem erwähnten Wendeschen Falle.

Sequeira. 17jähr. Taubstumme mit akutem Lupus erythe$\mathrm{m}$ a todes. Außer dem typischen schmetterlingsförmigen Gesichtsherd und düster roten Herden an den Ohrmuscheln finden sich an beiden Handrücken und Handgelenkstreckseiten mehrere livide Flecke ganz vom 
Aussehen eines toxischen Erythems. Keine subjektiven Symptome. Im Harne Eiweiß. Alle 15 Fälle dieser Form von Lupus erythematosus, die der Vortragende gesehen hat, betrafen junge. Weiber. Die Ãhnlichkeit mit Erythema multiforme weist auf toxische Ätiologie.

Diskussion. Little G. erinnert an zwei von ihm vorgestellte Frauen mit ganz ähnlichen Hautveränderungen. Whitfield berichtet über einen Mann, der einen kleinen Fleck von Lupus erythematodes im Gesichte hatte und im Anschluß an Typhus einen akuten Ausbruch bekam. P e r n et erwähnt, daß in einem seiner Fälle ein symmetrisches toxisches Exanthem bleibende Teleangiektasien zurückließ. Mac Leod berichtet über einen Fall ganz von der Art des oben vorgestellten.

Weber P. Lichen scrofulosorum vom Aussehen einer Psoriasis.

Whitfield. 1. Zwei Kulturen von Trichophyt on roseum, gezüchtet von einem Armherd einer Tinea circinata. Klinisch fiel die vollständig bullöse Beschaffenheit des ganzen Randes des Herdes auf.

2. Zwei leicht gelbbraune, sehr flache, nur leicht bestäubte Trichophyton-ectothrix-Kulturen von einem großen roten geschwollenen Tonsuranstleck des Haarbodens.

3. Präparat von der Hornschicht der Zehen, Trichophyton enthaltend. Bei dem P. trat der erste Herd der Pilzerkrankung vor 9 Jahren in inguine auf und kurz darauf ein Ekzem an den Zehen, das bis jetzt andauert. Der Reduer verweist auf seine Arbeiten über den Gegenstand and auf diejenigen $\mathrm{Sab}$ urauds (Epidermophyton inguinale) und wiederholt, daß die Infektion in einer vesikobullösen Dermatitis -Art akuten Ekzems oder Cheiropompholyx - oder aber in chronischer Schuppung, die gewöhnlich als "gichtisches" Ekzem diagnostiziert wird, zum Ausdrucke kommen kann.

Diskussion. Pernet hat mit Radcliffe-Crocker schon früher Pilzbefunde beim sogenannten Ekzem, besonders der Zehen, gemacbt, aber nicht veröffentlicht.

Williams, W. 1. Angi okeratom am kleinen Finger der einen

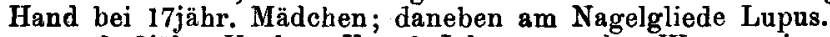

2. 8jähr. Knabe. Vor 3 Jahren an der Wange eine Vesikopustel wie ein Vakzinebläschen; nach der Heilung entwickelten sich in der Narbe und um sie allmählich $G$ ruppen von gat stecknadelkopf. groben ockergelben Erbabenheiten, die bei Glasdruck nur etwas opaker und gelber aussehen als Lupusknötchen. Keine Reaktion auf $5 \%$ ige Tuberkulinsalbe.

Diskussion. Whitfield diagnostiziert nicht Lupus, sondern eher Talgdrüsenbypertrophie, Little G. mit Wahrscheinlichkeit einen leichten Fall von Adenoma sebaceum Pringle.

3. Lupus des halben Haarbodens und fast des ganzen Gesichtes bei einem älteren Weibe, zum größten Teile spon+an geheilt mit weißer Narbe, die an diejenige eines Syphilids erinnert, zum anderen Teile durch sonnenlicht nach Eosinsensibilisierung und durch Röntgenbehandlung sehr gebessert.

(Ref. nach d. Originalberichte in Brit. Journ. Dermat. 1911. Nr. 5.)

Paul Sobotka (Prag). 